\title{
Leukocyte profile, gene expression, acute phase response, and metabolite status of cows with sole hemorrhages
}

\author{
Keelin O'Driscoll, ${ }^{* 1}$ Matthew McCabe, $\dagger$ and Bernadette Earley $†$ \\ *Pig Development Department, Animal and Grassland Research and Innovation Centre, Teagasc, Moorepark, Co. Cork, P61 C996, Ireland \\ †Animal and Bioscience Research Department, Animal and Grassland Research and Innovation Centre, Teagasc, Dunsany, Co. Meath, \\ C15 PW93, Ireland
}

\section{ABSTRACT}

Sole hemorrhages result from disruption to normal claw horn formation and are caused by a variety of internal and external factors. Evidence suggests that they are painful, although they do not usually cause clinical lameness and are difficult to detect by observing cow gait. Little is known about how or whether sole hemorrhages affect the cow systemically. This study compared hematology profile, leukocyte gene expression, and physiological responses of cows with no/mild hemorrhages (category $1 ; \mathrm{n}=17$ ), moderate hemorrhages (category $2 ; \mathrm{n}=18$ ), and severe hemorrhages (category $3 ; \mathrm{n}=12$ ). At approximately $100 \mathrm{~d}$ in milk, all cows in the study herd $(\mathrm{n}=374)$ were locomotion scored before hoof examination. The cows included in the study were not clinically lame and had no other hoof disorder. Blood samples were taken from all cows within $24 \mathrm{~h}$ of selection. Leukocyte counts were obtained using an automated cell counter, cortisol and dehydroepiandrosterone (DHEA) concentration by ELISA, and plasma haptoglobin, urea, total protein, creatine kinase and glucose were analyzed on a clinical chemistry analyzer. Expression of 16 genes associated with lameness or stress were estimated using real-time quantitative PCR. Data from cows within each category were compared using the Mixed procedure in SAS (version 9.3; SAS Institute Inc., Cary, NC). Fixed effects included hemorrhage severity category and lactation number, with days in milk and body condition score included as covariates. Locomotion score worsened as sole hemorrhage category worsened. Locomotion score of category 1 cows tended to be lower than that of category 2 cows and was lower than that of category 3 cows. The locomotion score of category 3 cows was also greater than that of categories 1 and 2 combined. Category had no effect on leukocyte number, on any of the individual leukocyte cell numbers or percentages, cor-

Received May 2, 2017

Accepted July 5, 2017.

${ }^{1}$ Corresponding author: keelin.odriscoll@teagasc.ie tisol or DHEA concentration, cortisol:DHEA ratio, or relative expression of any of the genes investigated, and we detected no differences in plasma glucose, protein, or creatine kinase concentrations between categories. However, category 3 cows had greater plasma concentrations of haptoglobin and tended to have lesser concentrations of plasma urea than category 1 and 2 cows. The differences in gait between cows with no or minor sole hemorrhages and cows with severe hemorrhages indicate that hemorrhages may be associated with discomfort or pain. Nevertheless, the only physiological measure that changed with increasing locomotion score was plasma haptoglobin concentration. Haptoglobin has previously been found to be elevated in lame cows, and thus shows promise as a marker for limb pain.

Key words: sole hemorrhage, immune, haptoglobin, locomotion

\section{INTRODUCTION}

Disruption to the formation of dairy cow hoof horn causes tissue damage that can result in a variety of claw horn disruption lesions. These include sole hemorrhages, which vary in severity from very faint yellow or pink patches of horn to extremely dark red areas. Sole hemorrhages are the visible evidence of disruption of blood flow to the corium, the part of the hoof responsible for synthesizing claw horn (Bicalho and Oikonomou, 2013). This disruption is thought to be caused by excessive movement of the third phalanx inside the hoof capsule, which in turn results from exposure to a variety of risk factors, such as hormonal changes around parturition, a thin digital cushion, long walking distances, or management in facilities with poor cow comfort (Bicalho and Oikonomou, 2013). Severe disruption to the corium can result in hoof horn damage to the extent that underlying tissue is exposed at the surface of the hoof, which is known as a sole ulcer. Thus, hemorrhages may be considered an early stage or minor version of sole ulcers (Bergsten, 2004).

Sole ulcers are one of the most severe hoof pathologies and are normally associated with clinical lameness 
(O'Driscoll et al., 2015). Clinical lameness has been demonstrated to be associated with a sickness response (Whay et al., 1997; Almeida et al., 2008), involving changes to both behavior and physiology and regulated by cytokines released by immune cells (Dantzer and Kelley, 2007). More specifically, O'Driscoll et al. (2015) found indications of systemic inflammation in cows that were lame only due to the presence of sole ulcers. For instance, cows with sole ulcers had a greater neutrophil: lymphocyte ratio, and greater concentrations of serum cortisol and dehydroepiandrosterone (DHEA) than cows without sole ulcers. Relative expression of several cytokine genes were more highly expressed in cows with ulcers than those without (IL1a, IL1,$C X C L 8$, and $I L 10$ ), as was relative expression of MMP13, GRa, $F A S, H P$, and CD62L. Increased expression of MMP13 mRNA has been found in cows with impaired locomotion (Almeida et al., 2007) and the gene could be implicated in development of claw horn disorders.

Although sole hemorrhages represent damage to the claw, the damage is not as severe as that caused by sole ulcers. Moreover, sole hemorrhages are not always associated with clinical lameness or indeed even easily detectable impairment to locomotory ability (Flower and Weary, 2006). Nevertheless, the underlying etiology for hemorrhages and ulcers is similar, and it is possible that hemorrhages are also associated with pain and immune system activation. Although it can be difficult to identify cows with sole hemorrhages by observing their gait (Flower and Weary, 2006), there is evidence of subclinical hoof pain in cows, which does not manifest in altered locomotion (Dyer et al., 2007). In the Dyer et al. (2007) study, the threshold of claw pain decreased nearly $30 \%$ below normal before there were any visible changes to locomotory ability. Pastell et al. (2010) reported that measurement of the ratio of weight applied to a pair of legs was reasonably able to discriminate between cows with or without sole hemorrhages, even though it was less successful at discriminating between cows with or without ulcers. Pastell et al. (2010) suggested that the level of pain associated with sole hemorrhages might vary depending upon the location on the foot or whether or not they were sole ulcers in the initial stage of development.

The objective of this study was to characterize the effects of sole hemorrhage severity on leukocyte profile, cortisol and DHEA response, metabolite profile, and expression of genes associated with lameness and stress. We hypothesized that increases in sole hemorrhage severity would be associated with increased expression of measures associated with lameness and activation of the immune system, even when the hemorrhages did not result in impaired gait. The results will contribute to knowledge regarding systemic effects of solar hemorrhaging and the severity of the disorder.

\section{MATERIALS AND METHODS}

All animal procedures performed in this study were conducted under experimental license (B100/4319) from the Irish Department of Health and Children in accordance with the Cruelty to Animals Act 1876 and the European Communities (Amendment of Cruelty to Animals Act 1876) Regulation 2002 and 2005. The study animals were located at the Teagasc Moorepark research farm, part of the Animal and Grassland Research and Innovation Centre (Teagasc, Moorepark, Fermoy, Co. Cork, Ireland). Samples were collected between April 2011 and February 2012. Laboratory analyses were carried out at the Animal and Bioscience Research Department, Animal and Grassland Research and Innovation Centre (Teagasc, Grange, Dunsany, Co. Meath, Ireland).

\section{Animals and Enrollment in the Study}

All cows from the Moorepark herd ( $\sim 375$ cows) were observed individually walking from the milking parlor after morning milking at approximately $100 \mathrm{~d}$ postpartum $[104 \pm 23$ (mean \pm SD) DIM; range 76 to 162]. They then had their hooves lifted and examined. Locomotion and hoof scoring was carried out by a single trained observer throughout the study. Only cows that were not clinically lame (defined as any obvious impairment to gait, including the cow being unwilling or slow to place one or more feet on the ground) but with sole hemorrhages of varying severity were used in the study. Individual cow records were examined using the herd recording system to ensure that the selected cows had remained free from health disorders during their current lactation. The final cohort included in the analysis consisted of 47 primiparous $(\mathrm{n}=14)$ and multiparous $(\mathrm{n}=33$; parity range 2 to 7 ; mean $3.5 \pm 1.3)$ HolsteinFriesian cows. Average milk yield, liveweight, and BCS [ranging from 1 (emaciated) to 5 (extremely fat) with increments of 0.25; Edmonson et al., 1989] at the time of taking experimental measures were $23.2 \pm 4.5 \mathrm{~kg} / \mathrm{d}$ per cow, $509 \pm 65 \mathrm{~kg}$, and $2.78 \pm 0.23$, respectively.

None of the enrolled cows were assigned to experimental treatments at the time of this study. The majority of the cows $(n=41)$ were enrolled while being kept on pasture during the summer (April to August 2011). These cows were fed a grass-based diet (approximately $80 \%$ perennial ryegrass) and managed using the "Moorepark" blueprint (McCarthy et al., 2013). The other 6 cows were enrolled during the winter housing 
period (December 2011 to February 2012), and were fed a TMR diet that included grass silage, maize silage, soy, and molasses. These cows were supplemented with $6 \mathrm{~kg}$ of concentrate per day in the milking parlor. It was hypothesized that the diets provided would not affect the immune status of the cows.

\section{Locomotion Scoring}

Cow locomotory ability was determined after morning milking as cows walked away from the milking parlor along a clean concrete track. They were observed from the side as they walked past and then from behind. Five aspects of locomotion were scored: spine curvature, tracking up, ab-/adduction, speed, and head bob, using the system adopted by O'Driscoll et al. (2010). Each aspect was scored from 1 to 5 , with 1 indicating no visible deviation from normal, and 5 being the worst possible score. These 5 aspects were summed to give one overall locomotion score per cow. Thus, the minimum score was 5 (perfect locomotion) and the maximum was 25 (extreme clinical lameness, cow virtually immobile). Cows were then held in a holding pen until their hooves were examined.

\section{Hoof Scoring}

Immediately after locomotion scoring, cows were restrained in a metal crate, their hind feet lifted and washed, and the claws individually examined for heel erosion, digital dermatitis, and white line disease (as described by O'Driscoll et al., 2008). Three cows had evidence of healed digital dermatitis lesions but were included because the lesions were not active. Due to the high frequency of other disorders, cows with superficial evidence of heel erosion $(\mathrm{n}=35)$ and white line disease $(\mathrm{n}=10)$ were also enrolled. The hind claws were then cleaned and a sliver of horn was trimmed using a quittor knife (Hauptner Quittor Hoof Knife, Channelle Vet Products, Loughrea, Ireland) from the entire area of the weight-bearing surface to expose fresh horn. Once the surface of the hoof was cleaned, each sole hemorrhage was given a score using the following scale, as per O'Driscoll et al. (2008): 1 = diffuse red, $2=$ stronger red coloration, $3=$ deep dense red, $4=$ port coloration, and $5=$ red raw, possibly fresh blood. Cows were placed into the following categories based upon the maximum sole hemorrhage score present on either hoof; category 1 : score $\leq 2$; category 2 : score $=3$; category 3 : score $>3$. In total, 17 cows were assigned to category 1 (no/mild hemorrhage present), 18 were assigned to category 2 (moderate hemorrhage present), and 12 were assigned to category 3 (severe hemorrhage present).

\section{Blood Sampling}

Blood samples were collected from each cow by jugular venipuncture the day after hoof examination. Blood samples were transported to the laboratory, stored at ambient temperature, and processed within $3.5 \mathrm{~h}$ for hematological analysis, or centrifuged, separated, and stored at $-20^{\circ} \mathrm{C}$ until cortisol, DHEA, and the other physiological assays were performed.

\section{Cortisol and DHEA}

Blood collected into a 9-mL serum separator tube (Vacutainer, Unitech Ltd., Dublin, Ireland) was used for measurement of cortisol and DHEA concentrations. Tubes were stored horizontally overnight at $4^{\circ} \mathrm{C}$, and then centrifuged at $1,000 \times g$ for $15 \mathrm{~min}$ at $4^{\circ} \mathrm{C}$. Serum was aspirated using a pipette and stored at $-20^{\circ} \mathrm{C}$ until analysis. Cortisol and DHEA concentrations were measured using ELISA (Enzo Life Sciences, Exeter, UK; Cortisol catalog no. ADI-901-071; DHEA catalog no. ADI-900-093). The sensitivity of the cortisol assay was $56.72 \mathrm{pg} / \mathrm{mL}$, and the intra- and interassay coefficients of variation based on controls were $<10.5$ and $<13.4 \%$, respectively. The sensitivity of the DHEA assay was $2.90 \mathrm{pg} / \mathrm{mL}$, and the intra- and interassay coefficients of variation based on controls were $<6.4$ and $<8.8 \%$, respectively.

\section{Physiological Variables}

Plasma haptoglobin $(\mathbf{H b})$, urea, total protein, creatine kinase, and glucose concentrations were analyzed using a clinical chemistry analyzer (Olympus AU 400, Tokyo, Japan) using reagents supplied by Olympus.

\section{Leukocyte Isolation from Whole Blood}

Thirty-six milliliters of blood collected into acidcitrate-dextrose (ACD) tubes from a subset of 19 animals (category $1, \mathrm{n}=9$; category $2, \mathrm{n}=7$, category $3, \mathrm{n}=3$, all multiparous) was pooled within $30 \mathrm{~min}$ of collection, according to the method of O'Loughlin et al. (2011). Briefly, red blood cells were lysed in a hypotonic solution followed by restoration in $12 \mathrm{~mL}$ of a hypertonic solution. The tubes were then centrifuged at $2,000 \times g$ to collect the leukocyte pellet, which was then washed twice with PBS, immediately frozen in liquid nitrogen, and stored at $-80^{\circ} \mathrm{C}$ until RNA extractions were performed. Pellets were resuspended in $1 \mathrm{~mL}$ of TRI reagent (Sigma-Aldrich Ireland Ltd., Dublin, Ireland), and pooled by animal immediately before further processing. 


\section{RNA Extraction and cDNA Synthesis}

A modified TRI reagent extraction method was used to extract total RNA from leukocytes via homogenization of the pellet in TRI reagent and the subsequent addition of chloroform followed by precipitation using isopropanol and ethanol. Then, RNA was quantified using a Nanodrop spectrophotometer (NanoDrop Technologies, Wilmington, DE). Quality of RNA was assessed on an Agilent 2100 Bioanalyser (Agilent Technologies Ireland Ltd., Dublin, Ireland) and only RNA samples with an RNA integrity number (RIN) $>7.0$ were used. Samples were treated with DNase and purified using an RNeasy mini kit (Qiagen Ltd., Crawley, UK). One microgram of total RNA per animal was reverse transcribed into cDNA using random hexamers and the High Capacity cDNA Reverse Transcription kit (Applied Biosystems, Dublin, Ireland) in a $20-\mu \mathrm{L}$ reaction and stored at $-20^{\circ} \mathrm{C}$.

\section{Real-Time Quantitative PCR}

Primers for candidate genes (Table 1) were designed based on bovine sequences obtained from the $\mathrm{Na}$ tional Center for Biotechnology Information database (https://www.ncbi.nlm.nih.gov/) using Primer Blast software (https://www.ncbi.nlm.nih.gov/tools/primer -blast/) and were commercially synthesized (SigmaAldrich Ireland Ltd., Dublin, Ireland). Amplified PCR products were sequenced (Macrogen Europe, Amsterdam-Zuidoost, the Netherlands) and confirmed, using BLAST (http://blast.ncbi.nlm.nih.gov/), to be identical to their respective bovine sequence. Amplification efficiencies were determined for all genes using serial dilutions of pooled cDNA samples. The formula $\mathrm{E}=$ $-1+10(-1 /$ slope $)$ was used where slope refers to the slope of the linear curve of cycle threshold $(\mathbf{C q})$ values plotted against $\log$ dilution. Only primers with PCR efficiencies between 90 and $130 \%$ were used in the current study.

Reference genes used in this study were selected based on previous experience within our research group (O'Loughlin et al., 2011; O'Driscoll et al., 2015). Three reference genes were used: $\beta$-actin $(A C T B)$, succinate dehydrogenase complex subunit A $(S D H A)$, and glyceraldehyde 3-phosphate dehydrogenase $(G A P D H)$. These were found to have a mean stability value, $\mathrm{M}$, of 0.51 . A normalization factor, calculated based on the geometric mean of the 3 reference genes, was used to normalize the expression of each gene of interest.

Real-time quantitative PCR (RT-qPCR) was performed using a 7500 Fast Real-Time PCR system
(Applied Biosystems). One microliter of cDNA was added to a $19-\mu \mathrm{L}$ master mix that included $10 \mu \mathrm{L}$ of Fast SYBR Green master mix (Applied Biosystems, Ireland), $8 \mu \mathrm{L}$ of nuclease-free water, and $0.5 \mu \mathrm{L}$ each of forward and reverse primers at concentrations individually optimized for each primer set. The following RT-qPCR cycle conditions were applied: $95^{\circ} \mathrm{C}$ for 20 $\mathrm{s}$ followed by 40 cycles of $95^{\circ} \mathrm{C}$ for $3 \mathrm{~s}$ and $60^{\circ} \mathrm{C}$ for $30 \mathrm{~s}$, finishing with amplicon dissociation at $95^{\circ} \mathrm{C}$ for $15 \mathrm{~s}, 60^{\circ} \mathrm{C}$ for $1 \mathrm{~min}$, increasing $0.5^{\circ} \mathrm{C}$ per cycle until $95^{\circ} \mathrm{C}$ was reached for $15 \mathrm{~s}$, followed by $60^{\circ} \mathrm{C}$ for $15 \mathrm{~s}$. In accordance with the MIQE guidelines (Minimum Information for Publication of Quantitative Real-Time PCR Experiments), raw $\mathrm{Cq}$ values were imported to GenEx Software v.5.2.2.8 (2010; MultiD Analyses AB, Göteborg, Sweden) and the following steps were performed: (1) outliers were removed from replicate wells using a modified Grubbs test at a $P<0.05$ confidence level for any replicate differing from the replicate mean by a standard deviation of more than 0.25 cycles; (2) the $\mathrm{Cq}$ values were corrected using the calculated efficiencies for each primer pair before determining the mean of replicates; (3) efficiency-corrected $\mathrm{Cq}$ values were then normalized to the reference genes; and (4) relative quantities (i.e., gene expression values relative to the highest $\mathrm{Cq}$ value) were calculated.

\section{Statistical Analyses}

Statistical analyses were carried out using SAS software (version 9.3, 2002; SAS Institute Inc., Cary, NC). All data were tested for normality before analysis by examination of boxplots and normal distribution plots. Residuals were examined to confirm normality of distribution for all data.

All data, including locomotion score, were analyzed using the Mixed procedure (SAS Institute Inc.). The model for locomotion and hoof scores, leukocyte data, and physiological assays included fixed effects of category $(1,2$, or 3$)$ and lactation number $(1$ or $>1)$; DIM and BCS were included as covariates. The score for each sole hemorrhage was geometrically adjusted to produce a severity score (sole hemorrhage scores of 1, 2, 3, 5, and 5 were adjusted to $1,2,4,8$, and 16 , respectively) to recognize the greater clinical significance of higher scores (Greenough and Vermunt, 1991). Hemorrhage scores for 4 claws were then summed to give a total sole hemorrhage score for each animal. These scores were compared between categories to determine whether the method of assigning cows to a category based upon the maximum score present reflected the overall level of hemorrhaging present. For analysis of gene expression, 
a similar model was used, but due to the low number of samples, analyzed cows from category $1(\mathrm{n}=9)$ were considered "mild" and those in categories $2(\mathrm{n}=7)$ and $3(\mathrm{n}=3)$ were combined into a single category called "severe." Lactation number was also removed because all cows were multiparous.

When significant effects were found, Tukey's test was used to establish pair-wise differences for fixed effects. Differences were considered significant at $P \leq 0.05$. Tendencies toward significance $(0.05<P \leq 0.10)$ are also presented.

\section{RESULTS}

\section{Locomotion and Hoof Scores}

Both category (Figure 1; $P<0.001$ ) and lactation number $(P<0.001)$ affected cow locomotion score. Locomotion score increased with category: cows in category 1 tended to have a lower locomotion score than those in categories $2(P=0.06)$ and $3(P<0.001)$. The score of category 3 cows was also greater than categories 1 and 2 combined $(P=0.05)$. Locomotion

Table 1. Gene, sequence, amplicon size and National Center for Biotechnology Information (NCBI) accession number of primers used to analyze gene expression by quantitative $\mathrm{PCR}^{1}$

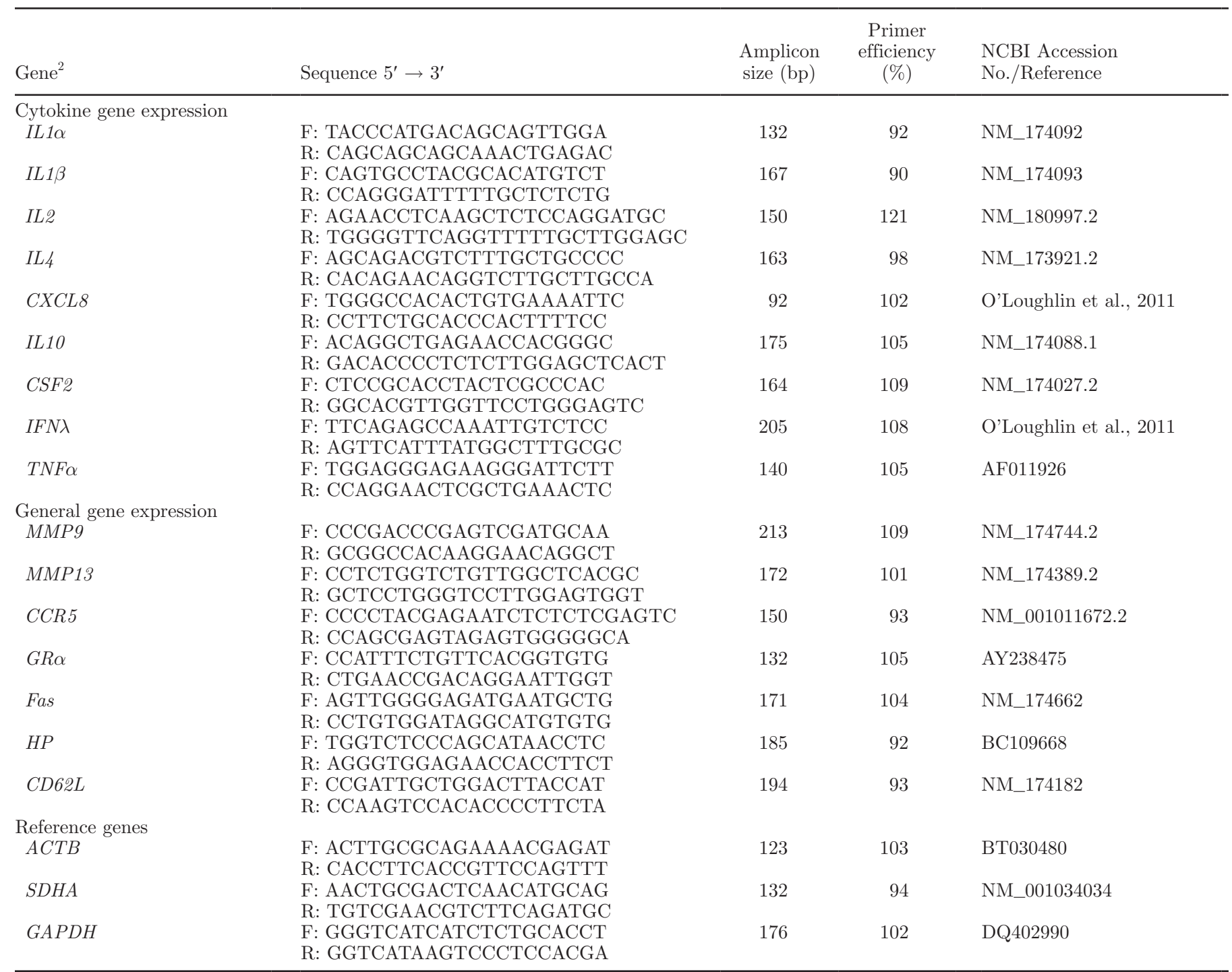

${ }^{1}$ The primers for quantitative PCR candidate genes were obtained using Primer Blast software (https://www.ncbi.nlm.nih.gov/tools/primerblast/) and based on bovine sequences obtained from the NCBI database (https://www.ncbi.nlm.nih.gov/); $\mathrm{F}=$ forward; $\mathrm{R}=$ reverse.

${ }^{2} C X C L 8=\mathrm{IL}-8, C S F 2=$ colony stimulating factor-2, TNF $\alpha=$ tumor necrosis factor $\alpha, M M P 9=$ matrix metallopeptidase-9, $M M P 13=$ matrix metallopeptidase-13, CCR $5=\mathrm{C}$ C chemokine receptor-5, GR $\alpha=$ glucocorticoid receptor $\alpha, H P=$ haptoglobin; $C D 62 L=\mathrm{L}-\mathrm{selectin}, A C T B=$ $\beta$-actin, $S D H A=$ succinate dehydrogenase complex subunit A. 
score was lower for cows in their first lactation $(5.6 \pm$ $0.4)$ than for those in subsequent lactations $(7.6 \pm 0.2$; $P<0.001)$. We detected an effect of category on total sole hemorrhage score (Figure 1B; $P<0.001$ ); there was a significant difference between all categories at $P$ $<0.001$.

\section{Leukocyte Population and Hematological Analysis}

Category did not affect leukocyte number or any of the individual leukocyte cell numbers or percentages (Table 2).

\section{Cortisol and DHEA}

There was no effect of category on cortisol or DHEA concentration. However, cortisol concentration increased numerically with category and DHEA decreased; cows in category 3 tended to have lower DHEA concentrations than cows in the other 2 categories (Figure 2; $P$ $=0.1$ ). Likewise, category did not affect cortisol:DHEA ratio, but the ratio increased numerically with category.

\section{Haptoglobin, Glucose, Protein, Urea, and Creatine Kinase}

Category affected haptoglobin level (Table 3); cows in category 3 had significantly greater plasma concentrations than cows in category $1(P=0.05)$, with cows in category 2 being intermediate. Plasma haptoglobin concentrations were also higher in cows in category 3 than in categories 1 and $2(P<0.05)$, and cows in category 1 tended to have lower haptoglobin concentrations than those in categories 2 and $3(P=0.08)$.

Plasma glucose and total protein concentrations did not differ across categories, although concentrations of both increased numerically as the severity of hemorrhaging increased. Although there was no overall effect of category on plasma urea concentrations, cows in category 3 tended to have greater concentrations than those in categories 1 and $2(P=0.1)$. Likewise, we detected no difference in protein:urea ratio across categories, but category 1 cows tended to have a greater protein:urea ratio than those in the other categories $(P=0.09)$. There was no effect of category on plasma creatine kinase activity.

\section{Gene Expression}

Of the 9 cytokine genes investigated, none were differentially expressed between the mild and severe categories (Figure 3 ). We detected no effect of category on expression of MMP9, MMP13, CCR5, GR $\alpha, F A S, H P$, or CD62L genes.
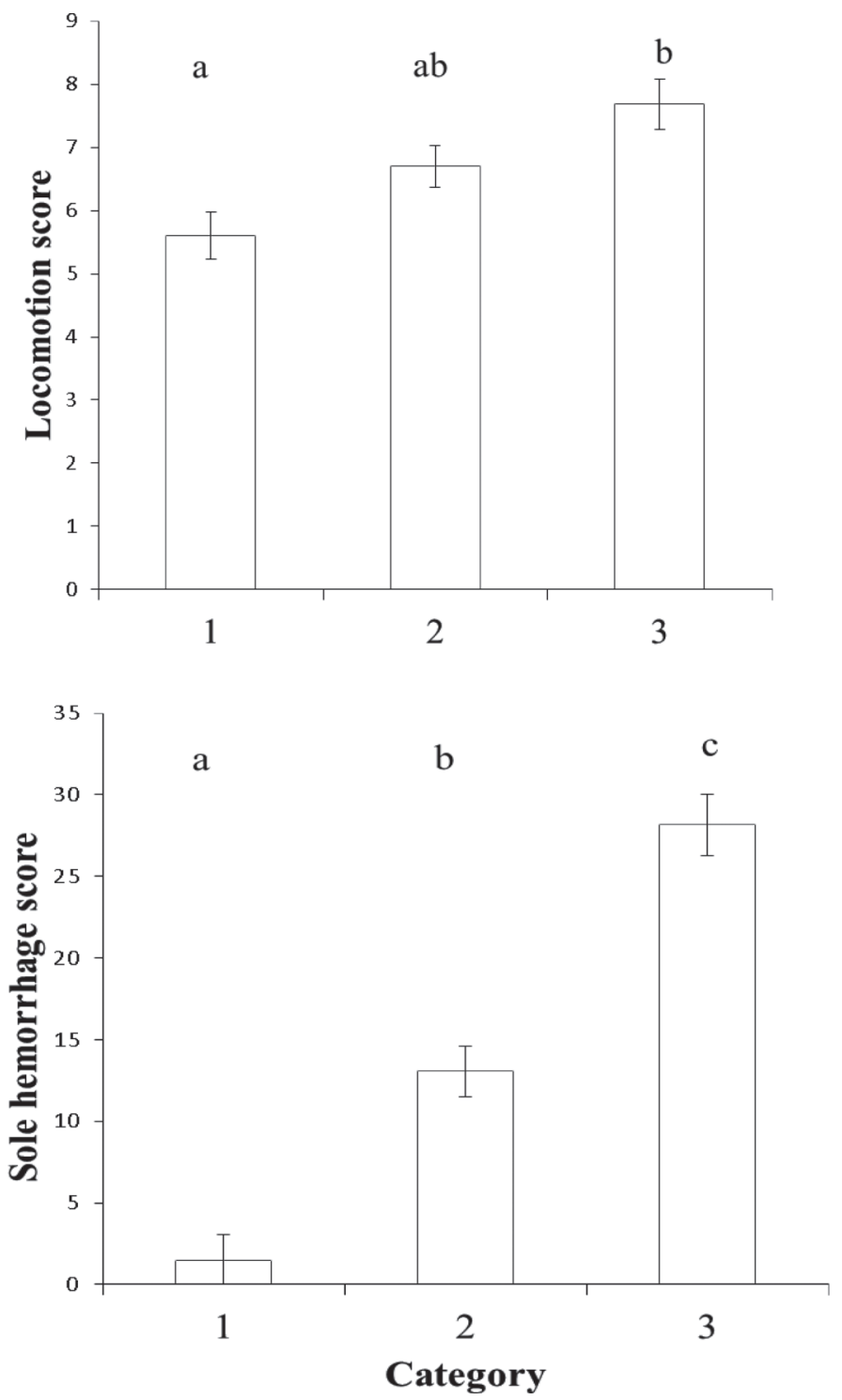

Figure 1. Locomotion scores (top) and overall sole hemorrhage scores (bottom) of cows in each category. Category 1 was defined as having a maximum sole hemorrhage score of 2 ; category 2 as having a score of 3 ; and category 3 as having a maximum score of 4 or 5 . Data are presented as least squares means and standard errors. Letters $(\mathrm{a}-\mathrm{c})$ indicate differences between categories at $P<0.05$.

\section{DISCUSSION}

Several studies have demonstrated an association between behavioral expression of lameness in dairy cows, and activation of aspects of the immune system (Almeida et al., 2007, 2008; O'Driscoll et al., 2009, 2015). However, less work has been carried out to investigate the relationship between the immune system and specific individual lameness-causing pathologies. Moreover, this study is the first, to our knowledge, to investigate whether a disorder of the hoof theorized to be painful 


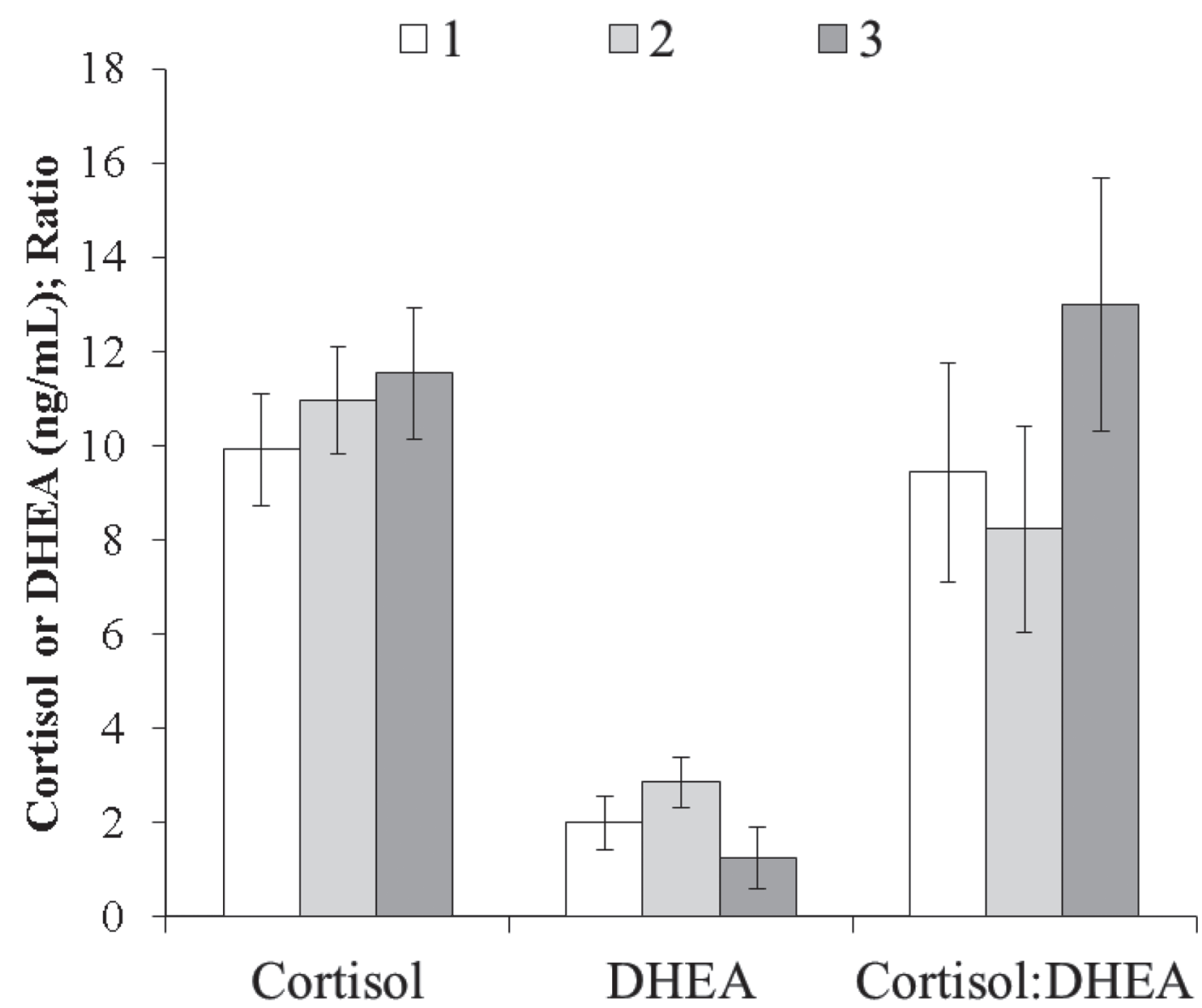

Figure 2. Serum cortisol and dehydroepiandrosterone (DHEA) concentrations in cows with varying levels of sole hemorrhage (1, 2 and 3 ). Category 1 was defined as having a maximum sole hemorrhage score of 2; category 2 as having a score of 3 ; and category 3 as having a maximum score of 4 or 5 . Data are presented as least squares means and standard errors.

but not severe enough to cause clinical lameness is associated with a systemic physiological response by the animal.

A significant challenge in this study was to identify animals with sole hemorrhages and no other disorder. During the study, 374 animals were screened for the presence of hemorrhages, but only 37 cows were considered appropriate for inclusion, even allowing for presence of superficial white line disease and heel erosion. We included cows with superficial levels of these 2 disorders because they arise from external mechanical insults to the hoof (e.g., standing on damp surfaces or

Table 2. Total leukocyte, neutrophil, and lymphocyte counts $\left(\times 10^{3}\right.$ cells $\left./ \mu \mathrm{L}\right)$; neutrophil:lymphocyte $(\mathrm{N}: \mathrm{L})$ ratio; and eosinophil and monocyte counts $\left(\times 10^{3}\right.$ cells $\left./ \mu \mathrm{L}\right)$, and percentages for cows in each category $(1,2, \text { or } 3)^{1,2}$

\begin{tabular}{|c|c|c|c|c|c|c|c|c|}
\hline \multirow[b]{2}{*}{ Variable } & \multicolumn{4}{|c|}{ Count } & \multicolumn{4}{|c|}{ Percentage } \\
\hline & 1 & 2 & 3 & $P$-value & 1 & 2 & 3 & $P$-value \\
\hline Neutrophils & $3.21 \pm 0.32$ & $3.18 \pm 0.33$ & $3.53 \pm 0.40$ & 0.78 & $36.0 \pm 2.1$ & $35.4 \pm 1.9$ & $37.6 \pm 2.2$ & 0.75 \\
\hline Lymphocytes & $4.22 \pm 0.32$ & $4.41 \pm 0.28$ & $4.20 \pm 0.33$ & 0.85 & $49.4 \pm 2.2$ & $50.5 \pm 2.0$ & $47.8 \pm 2.3$ & 0.66 \\
\hline $\mathrm{N}: \mathrm{L}$ & $0.73 \pm 0.10$ & $0.70 \pm 0.09$ & $0.85 \pm 0.11$ & 0.56 & NA & NA & & \\
\hline Eosinophils & $0.62 \pm 0.10$ & $0.65 \pm 0.09$ & $0.63 \pm 0.10$ & 0.92 & $7.5 \pm 1.1$ & $7.2 \pm 1.0$ & $7.0 \pm 1.1$ & 0.95 \\
\hline
\end{tabular}

${ }^{1}$ Category 1 was defined as having a maximum sole hemorrhage score of 2 ; category 2 as having a score of 3; and category 3 as having a maximum score of 4 or 5 .

${ }^{2}$ Values are shown $\pm \mathrm{SE}$.

${ }^{3} \mathrm{NA}=$ not applicable. 


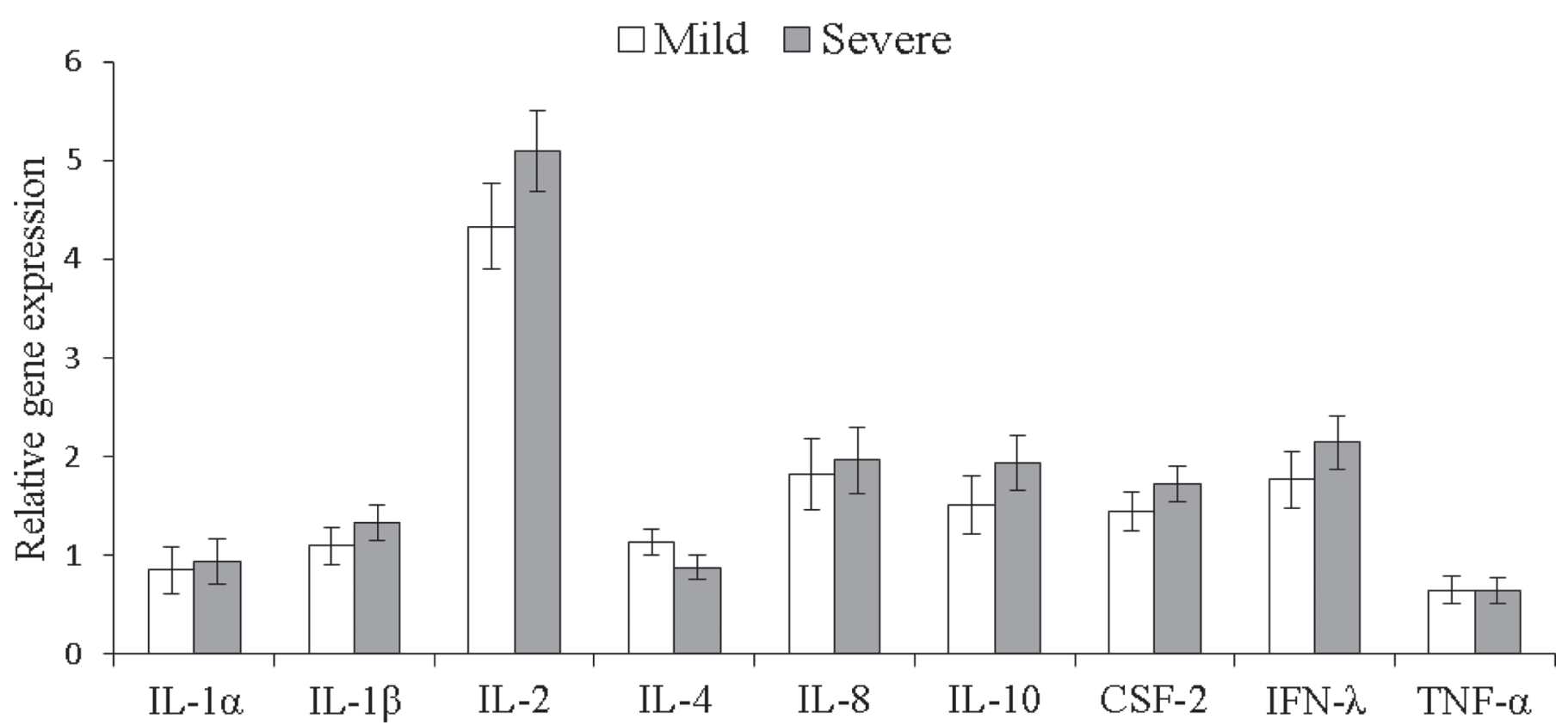

Figure 3. Relative gene expression of cows in each category. Values were normalized to reference genes followed by calculation of relative quantities to the highest cycle threshold value. Cows in the mild category had a maximum sole hemorrhage score of 2 , and those in the severe category had a minimum score of 3. CSF-2 $=$ colony stimulating factor-2, $T N F-\alpha=$ tumor necrosis factor- $\alpha$. Data are presented as least squares means and standard errors.

exposure to stones while walking to the parlor) rather than resulting from an infectious agent or a metabolic or physiological problem. At the mild or preliminary stages of the disorders, we hypothesize that they may not be noticed by the cow, as the damage to horn is shallow. Cows in pasture-based systems are prone to development of white line disease and heel erosion due to the nature of the system (long distance to the parlor, exposure to wet grass). Thus, if similar work were to be carried out in the future, an indoor-housed herd may yield a greater number of cows suitable for inclusion.

Unlike previous studies, we found that locomotion scores differed between cows with no or minor sole hemorrhages and cows with severe hemorrhages. For instance, Flower and Weary (2006) found that neither a visual analog scale nor a 5-point scoring system discriminated between cows with and without sole hemorrhages. More recently, Tadich et al. (2010) found the same, even though, in both of these studies, cows with or without sole ulcers displayed differences in gait. This could be due in part to the level of detail of the locomotion scoring system we used. Both of the previously cited studies used numerical rating systems, which scored cows from 0 (normal) to 5 (severely lame). However, our system scored 5 individual aspects of locomotion from 0 to 5 , which provides an additional layer of detail. The authors of the current study were blind to hemorrhage score (or any other condition) at the time of locomotion scoring, as the cows' feet had not yet been examined, which adds validity to the results. It is

Table 3. Metabolite levels for cows in each category

\begin{tabular}{lcccc}
\hline & \multicolumn{3}{c}{ Sole hemorrhage category $^{1}$} \\
\cline { 2 - 4 } Variable & 1 & 2 & 3 & $P$-value \\
\hline Haptoglobin $(\mathrm{mg} / \mathrm{mL})$ & $1.36 \pm 0.25^{\mathrm{a}}$ & $1.54 \pm 0.22^{\mathrm{a}}$ & $2.20 \pm 0.26^{\mathrm{b}}$ & 0.05 \\
Glucose $(\mathrm{mmol} / \mathrm{L})$ & $3.32 \pm 0.07$ & $3.36 \pm 0.07$ & $3.36 \pm 0.09$ & 0.92 \\
Protein $(\mathrm{g} / \mathrm{L})$ & $83.6 \pm 1.5$ & $85.1 \pm 1.3$ & $85.3 \pm 1.6$ & 0.69 \\
Urea $(\mathrm{mmol} / \mathrm{L})$ & $6.12 \pm 0.56$ & $6.10 \pm 0.50$ & $5.03 \pm 0.59$ & 0.31 \\
Creatinine kinase $(\mu \mathrm{mol} / \mathrm{L})$ & $169.4 \pm 10.9$ & $172.5 \pm 11.6$ & $180.5 \pm 13.0$ & 0.80 \\
Urea:creatinine $(\mathrm{mmol} / \mu \mathrm{mol})$ & $0.040 \pm 0.004$ & $0.033 \pm 0.004$ & $0.030 \pm 0.004$ & 0.24 \\
\hline
\end{tabular}

${ }^{\mathrm{a}, \mathrm{b}}$ Different superscripts indicate a significant difference $(P<0.05)$.

${ }^{1}$ Category 1 was defined as having a maximum sole hemorrhage score of 2; category 2 as having a score of 3 ; and category 3 as having a maximum score of 4 or 5 . 
important to note that locomotion scores differed between sole hemorrhage categories in the direction that would be expected if they caused pain.

A second reason why differences in locomotory ability may have been detected in this study but not in other studies is that, in general, other experiments simply classified cows based on the presence or absence of hemorrhages. Abnormalities of gait are associated more with the severity rather than the size of lesions (Whay et al., 1997), which is why we decided to categorize cows based upon the worst hemorrhage score present rather than number of hemorrhages or area covered. An analysis of overall scores was carried out to determine whether this method reflected the level of severity of hemorrhaging on the hoof, as estimated in previously published work (O'Driscoll et al., 2008, 2010). The significant differences between the cohorts of cows with regard to total hemorrhage scores provided reassurance regarding the separation of the groups.

Nevertheless, we found no effect of sole hemorrhage category on any aspect of the leukogram, expression of the genes investigated, or on most of the metabolites. Although O'Driscoll et al. (2015) found that cows that were clinically lame because of sole ulcers had a leukocyte profile indicative of systemic inflammation and stress, this pattern was not evident in the current study. It is possible that only claw horn disorders severe enough to cause clinical lameness, and thus a sickness response, cause a systemic response. Moreover, many of the variables in that previous study only tended to be different, and thus our results are perhaps not surprising. However, in general, the numerical differences in the variables measured were in the same direction as the significant differences detected in the previous experiment (O'Driscoll et al., 2015). For instance, cortisol concentration and cortisol:DHEA ratio increased numerically with increasing severity of sole hemorrhage. Although a larger sample size would likely have yielded more statistical differences, it is important to note that in no instance did the levels of the parameters measured fall outside the normal range for cattle. Moreover, the variances around the means calculated for measures in this study in general were not greater than in our previous study that investigated differences between sound cows and cows with sole ulcers (O'Driscoll et al., 2015). Thus, although a larger sample size may have yielded statistical differences, the biological significance would probably have been low. Considering this, it appears that sole hemorrhages, although likely to cause a measure of pain, do not stimulate a systemic response.

Previous work with lame cows and cows with sole ulcers has also shown elevated plasma haptoglobin concentrations in affected cows (Jawor et al., 2008; Smith et al., 2010; O'Driscoll et al., 2015). The current study is the first to demonstrate that haptoglobin concentration is also elevated in cows with hemorrhages that are not clinically lame. Tadich et al. (2013) found that haptoglobin concentration was elevated in cows with a locomotion score greater than 1 , using a 5 -point scale where 1 is the best possible locomotion and 5 indicates severe lameness. Those authors also measured level of hyperalgesia and concluded that haptoglobin concentration can be a sensitive measure of pain from lameness. These results are in agreement with the results from our study, whereby increases in haptoglobin concentration across hemorrhage severity category were parallel to increases in locomotion score, even though none of the cows were clinically lame. Thus, haptoglobin concentrations show promise as a marker for painful claw horn disorders.

The second potentially useful marker identified was urea: we detected a tendency for urea concentrations to be lower in cows in the most severe hemorrhage category than in cows in the other 2 categories. O'Driscoll et al. (2015) reported that cows with sole ulcers had lower urea concentrations than sound cows. There is little published literature regarding a potential link of urea concentrations with lameness in cows. However, serum urea concentrations have been observed to be lower in sheep and beef cattle that are submitted to stressors such as isolation, restraint, and transportation (Galyean et al., 1981; Apple et al., 1993).

\section{CONCLUSIONS}

Although the locomotion scoring system used in this study was able to distinguish between cows with no or mild, moderate, and severe hemorrhages, these differences were not reflected in the majority of the physiological measures evaluated. Haptoglobin concentrations were elevated in cows with severe hemorrhaging, which is consistent with previous research. However, the level of tissue damage associated with sole hemorrhaging was probably not severe enough to stimulate a generalized activation of the immune system.

\section{ACKNOWLEDGMENTS}

The authors gratefully acknowledge the assistance of John Paul Murphy and Aidan Brennan (both from Teagasc, Moorepark, Fermoy, Co. Cork, Ireland) for their assistance in screening animals for inclusion in the study, and Margaret Murray (Teagasc, Dunsany, Grange, Co. Meath, Ireland) for hematology analysis. This study was funded by a Marie Curie Intra-European Fellowship (FP7-People 2009-IEF; Grant agreement number: 252611) to Keelin O'Driscoll. 


\section{REFERENCES}

Almeida, P. E., P. S. D. Weber, J. L. Burton, R. J. Tempelman, J. P. Steiber, and A. J. Zanella. 2007. Gene expression profiling of peripheral mononuclear cells in lame dairy cows with foot lesions. Vet. Immunol. Immunopathol. 120:234-245. https://doi.org/10 $.1016 /$ j.vetimm.2007.06.028.

Almeida, P. E., P. S. D. Weber, J. L. Burton, and A. J. Zanella. 2008. Depressed DHEA and increased sickness response behaviors in lame dairy cows with inflammatory foot lesions. Domest. Anim. Endocrinol. 34:89-99. https://doi.org/10.1016/j.domaniend.2006 .11 .006 .

Apple, J. K., J. E. Minton, K. M. Parsons, and J. A. Unruh. 1993. Influence of repeated restraint and isolation stress and electrolyte administration on pituitary-adrenal secretions, electrolytes, and other blood constituents of sheep. J. Anim. Sci. 71:71-77.

Bergsten, C. 2004. Causes, risk factors, and prevention of laminitis and related claw lesions. Acta Vet. Scand. 44(Suppl. 1):157-166. https://doi.org/10.1186/1751-0147-44-S1-S157.

Bicalho, R. C., and G. Oikonomou. 2013. Control and prevention of lameness associated with claw lesions in dairy cows. Livest. Sci. 156:96-105. https://doi.org/10.1016/j.livsci.2013.06.007.

Dantzer, R., and W. W. Kelley. 2007. Twenty years of research on cytokine-induced sickness behaviour. Brain Behav. Immun. 21:153160.

Dyer, R. M., N. K. Neerchal, U. Tasch, Y. Wu, P. Dyer, and P. G. Rajkondawar. 2007. Objective determination of claw pain and its relationship to limb locomotion score in dairy cattle. J. Dairy Sci. 90:4592-4602. https://doi.org/10.3168/jds.2007-0006.

Edmonson, A. J., I. J. Lean, L. D. Weaver, T. Farver, and G. Webster. 1989. A body condition scoring chart for Holstein dairy cows. J. Dairy Sci. 72:68-78. https://doi.org/10.3168/jds.S0022 -0302(89)79081-0.

Flower, F. C., and D. M. Weary. 2006. Effect of hoof pathologies on subjective assessments of dairy cow gait. J. Dairy Sci. 89:139-146. https://doi.org/10.3168/jds.S0022-0302(06)72077-X.

Galyean, M. L., R. W. Lee, and M. E. Hubbert. 1981. Influence of fasting and transit on ruminal and blood metabolites in beef steers. J. Anim. Sci. 53:7-18. https://doi.org/10.2527/jas1981.5317.

Greenough, P. R., and J. J. Vermunt. 1991. Evaluation of subclinical laminitis in a dairy herd and observations on associated nutritional and management factors. Vet. Rec. 128:11-17. https://doi.org/10 $.1136 / v r .128 .1 .11$

Jawor, P., S. Steiner, T. Stefaniak, W. Baumgartner, and A. Rzasa. 2008. Determination of selected acute phase proteins during the treatment of limb diseases in dairy cows. Vet. Med. Czech. 53:173-183.

McCarthy, B., K. M. Pierce, L. Delaby, A. Brennan, C. Fleming, and B. Horan. 2013. The effect of stocking rate and calving date on grass production, utilization and nutritive value of the sward during the grazing season. Grass Forage Sci. 68:364-377.

O'Driscoll, K., L. Boyle, P. French, and A. Hanlon. 2008. The effect of out-wintering pad design on hoof health and locomotion score of dairy cows. J. Dairy Sci. 91:544-553. https://doi.org/10.3168/ jds.2007-0667.

O'Driscoll, K., M. McCabe, and B. Earley. 2015. Differences in leukocyte profile, gene expression, and metabolite status of dairy cows with or without sole ulcers. J. Dairy Sci. 98:1685-1695. https://doi .org/10.3168/jds.2014-8199.

O'Driscoll, K., B. O'Brien, D. Gleeson, and L. Boyle. 2010. Effect of milking frequency and nutritional level on hoof health, locomotion score and lying behaviour of dairy cows. Livest. Sci. 127:248-256. https://doi.org/10.1016/j.livsci.2009.10.006.

O'Driscoll, K. K. M., A. C. Lossie, M. M. Schutz, and S. D. Eicher. 2009. The effect of floor surface on dairy cow immune function and locomotion score. J. Dairy Sci. 92:4249-4261. https://doi.org/10 $.3168 /$ jds.2008-1906.

O'Loughlin, A., M. McGee, S. M. Waters, S. Doyle, and B. Earley 2011. Examination of the bovine leukocyte environment using immunogenic biomarkers to assess immunocompetence following exposure to weaning stress. BMC Vet. Res. 7:45.

Pastell, M., L. Hänninen, A. M. de Passillé, and J. Rushen. 2010. Measures of weight distribution of dairy cows to detect lameness and the presence of hoof lesions. J. Dairy Sci. 93:954-960. https://doi $.0 r g / 10.3168 /$ jds.2009-2385.

Smith, B. I., J. Kauffold, and L. Sherman. 2010. Serum haptoglobin concentrations in dairy cattle with lameness due to claw disorders. Vet. J. 186:162-165. https://doi.org/10.1016/j.tvj1.2009.08.012.

Tadich, N., S. Bastias, C. Rosenfeld, and L. E. Green. 2013. Nociceptive threshold, blood constituents and physiological values in 213 cows with locomotion scores ranging from normal to severely lame. Vet. J. 197:401-405. https://doi.org/10.1016/j.tvj1.2013.01.029.

Tadich, N., E. Flor, and L. Green. 2010. Associations between hoof lesions and locomotion score in 1098 unsound dairy cows. Vet. J. 184:60-65. https://doi.org/10.1016/j.tvjl.2009.01.005.

Whay, H. R., A. E. Watermann, and A. J. Webster. 1997. Association between locomotion, claw lesions and nociceptive threshold in dairy heifers during the prepartum period. Vet. J. 154:155-161. https://doi.org/10.1016/S1090-0233(97)80053-6. 